\title{
Multifrequency Bioimpedance Phase Angle as a Tool to Predict Maturation Failure of Native Fistulae for Hemodialysis
}

\author{
José Lucas Daza ${ }^{1, *}$, John F Galindo², Mónica R Villaquiran ${ }^{3}$, Emilio D Valenzuela ${ }^{4}$, Andres Cardenas ${ }^{5}$, Luis Jose Daza ${ }^{6}$, Maria \\ Camila Correcha7, Jonathan de Jong ${ }^{7}$, Yaroslad De La Cruz Prieto ${ }^{8}$, Gerardo Gutierrez ${ }^{9}$, and Luis Puello ${ }^{10}$
}

\author{
${ }^{2}$ Department of General Medicine, Ibague, Colombia \\ ${ }^{3}$ Department of Nutrition Ibague, Colombia \\ ${ }^{4}$ Intensive Care Unit, Santiago, Chile \\ ${ }^{5}$ Medical Student, Santa Marta, Colombia \\ ${ }^{6}$ Department of Hematology, Cartagena, Colombia \\ ${ }^{7}$ Medical Student, Manizales, Colombia \\ ${ }^{8}$ Department of Nephrology, Cali, Colombia \\ ${ }^{9}$ Department of Nephrology, Villavicencio, Colombia \\ ${ }^{10}$ Department of Nephrology, Cartagena, Colombia
}

${ }^{1}$ Nephrology and Dialysis Unit Fresenius Medical Care, Ibagué, Colombia

*Corresponding author: José Lucas Daza, Internist, Nephrology and Dialysis Unit Fresenius Medical Care, Ibagué, Barrio Restrepo Macarena Alta Calle 41 \#5-40, Ibagué 73001, Colombia, E-mail: drlucasdaza@gmail.com

Received: 24 Jun, 2021 | Accepted: 09 Jul, 2021 | Published: 19 Jul, 2021

Citation: Daza JL, Galindo JF, Villaquiran MR, Valenzuela ED, Cardenas A, et al. (2021) Multifrequency Bioimpedance Phase Angle as a Tool to Predict Maturation Failure of Native Fistulae for Hemodialysis. Int J Nephrol Kidney Fail 7(3): dx.doi.org/10.16966/2380-5498.215

Copyright: (c) 2021 Daza JL, et al. This is an open-access article distributed under the terms of the Creative Commons Attribution License, which permits unrestricted use, distribution, and reproduction in any medium, provided the original author and source are credited.

\begin{abstract}
Background and objectives: An arteriovenous fistula is considered to be an ideal vascular access for patients receiving hemodialysis, its main limitation is its high failure rate to achieve maturation and long-term functionality loss. Multiple strategies have attempted to identify patients at risk. Bioelectrical impedance has shown to be a valuable resource in the determination of the hydration status, and the measurement of the phase angle through this method has demonstrated to be a good indicator of the nutritional state and it's related as a general marker of survival. The objective of this study is to analyze the role of plasma albumin and phase angle measured through bioelectrical impedance as tools useful for predicting failure of arteriovenous fistulas.
\end{abstract}

Materials and methods: Prospective observational study, including 104 patients with chronic kidney disease receiving hemodialysis that underwent a native arteriovenous fistula during a period of 24 months. Analyzing its clinical characteristics, laboratory and phase angle through electrical bioimpedance, both univariate and multivariate analysis was performed both with logistic regression, furthermore calculation of coordinates and ROC curve to establish a better cut-off point.

Results: Variables that were analyzed only the phase angle measurement showed statistical significance OR 2.61 (1.6-4.4) P-value 0.001 for predicting arteriovenous fistula failure. In female patients with a phase angle value of 3.25 had a $90 \%$ sensibility and $53 \%$ specificity for male patients a value of 3.58 showed a sensibility of $84 \%$ and a specificity of $52 \%$ with ROC curve of 0.78 .

Conclusion: Phase angle through bioimpedance is a useful parameter, helpful in predicting failure of native arteriovenous fistula, it is also an important tool for identifying patients at risk, in early stages prior to the construction of a vascular access.

Keywords: Chronic kidney disease; Arteriovenous fistula; Phase angle; Multi-frequency bioimpedance

\section{Introduction}

Native arteriovenous fistulas are considered to this date to be the vascular access of choice widely recommended to patients undergoing chronic hemodialysis, due to its advantages, such as its cost-benefit relation, longer duration, lower rate of infection, hospital admission and mortality $[1,2]$.

However, its main inconvenience, is the failure or loss of the vascular access, depending on the series this can be around $60 \%$ in the first and includes primary failure presenting within the first 72 hours after its constructions as well as loss of functionality which is described as an impossibility of achieving an adequate dialytic treatment after 6 or 8 weeks with maturation two needle techniques, representing an important cause of morbidity, hospital admission and an increase in healthcare costs [3-5].

Various factors have been identified to this date related with the failure of establishing a vascular access, some in a clearer manner than other; however none of these factors has shown a relevant role in an isolated manner [6-8]. 
Within the multiples factors identified and those which possible adverse association with an arteriovenous fistula is still unknown among these is the nutritional status $[9,10]$, currently known as protein-energy wasting syndrome, its association with cardiovascular risk and mortality in patients undergoing hemodialysis $[9,11,12]$. The relation between malnutriton and adverse surgery results in terms of complications, days of hospital stay, infection and mortality; [13] however its association with adverse results in arteriovenous fistulas has not been demonstrated, partially due to difficulties in defining its criteria, among the tools used for its quantification and the use of biological and biochemical markers such as albumin which presents important limitations in patients undergoing hemodialysis.

Recently, electrical bioelectrical impedance has become a valuable tool in the area of nephrology; this is based on the fact that biological systems due to their composition of electrolytes and cellular membranes with specific polarities are considered conductors of electrical current $[14,15]$. Bioelectrical impedance is a technique which determines the opposition of an organism as an alternating current passes through, giving a measurement of impedance $(Z)$ which is the result of two components: the resistance presented when a current passes through which is represented by the content of body water and its reactance which reflects the capacity of cells to store electrical energy (serving as condensers) in theory this correlates with the integrity of a cellular membrane. The two components, the resistance (R) which reflects the hydration status and the reactance $(\mathrm{Xc})$ which indicates the nutritional status are represented as a vector and reflects the body water and cellular integrity respectively; the angle resulting from these two measurements is known as the phase angle (PA) which in eutrophic patients with no nutritional deficit has a value inferior to 10 degrees and has been consistently related with nutritional status [16-19].

Bioimpedance allows the calculation of body composition in terms of percentages of fat tissue and lean tissue, its hydration status in terms of a percentage of hydration in relation to intra-and extracellular liquid, which has allowed for a more precise quantification of nutritional status and dry weight [18-20].

The phase angle is considered to be a measurement of cellular stability and integrity that has a well-defined correlation with nutritional status and is considered to be an important predictor of survival in patients with chronic kidney disease compared with other traditionally used determinations such as DEXA (Dual energy X-ray absorptiometry assessment), global subjective evaluation and biochemical markers like total proteins and albumin; and its relation with mortality in this population has been described [21-23] (Figure 1).

To this date only few studies have taken into account the relation between plasma albumin levels and adverse results related to arteriovenous fistulas, suggesting that low levels of plasma albumin could associate with failure of maturation of native fistulas [24,25]; however, considering that albumin is not an ideal marker for evaluating nutritional status in patients undergoing hemodialysis, its role as a tool helpful in predicting the probability of success or failure of a vascular access like an arteriovenous fistula is still unclear.

The objective of this study is to evaluate the performance of plasma albumin, phase angle through multi-frequency bioimpedance and its value as a potential predictor of failure in arteriovenous fistulas for hemodialysis and establish if malnutrition determined through this behaves as a new risk factor for adverse results related to vascular access.

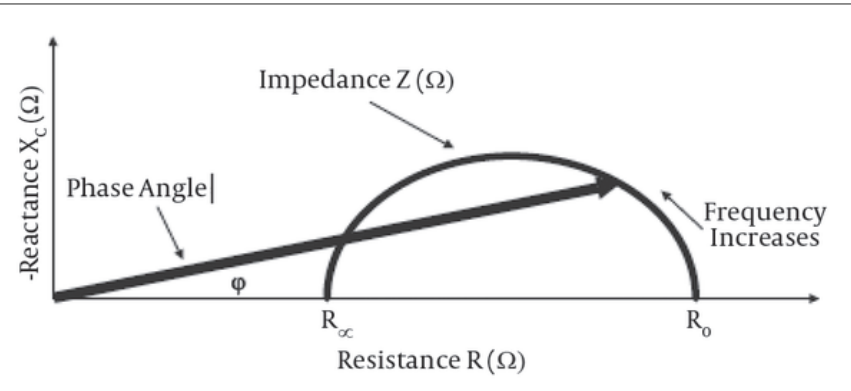

Figure 1: Graphic derivation diagram of the phase angle and its relation to resistance $(R)$, reactance $(X c)$, impedance $(Z)$ and frequency of the current applied.

\section{Baseline patients characteristics}

Initially, 109 patients from a single colombian dialysis center who were subjected to native FAV construction at the discretion of the vascular surgeon of the dialysis center were recruited; both proximal and distal fistulas were included during de follow-up period and met the inclusion criteria. The information from five patients was not included in the final analysis, a patient carrying pacemaker who did not allow reliable bio impedance data, four lost, 3 cardiovascular deaths and a loss of tracking. Finally, 104 patients were included for the corresponding statistical analysis. It is not possible to establish comparison groups as to date there is not a gold standard reference test for vascular access failure detection at the pre-operative stage (Figure 2).

\section{Materials and Methods}

An observational prospective study was realized during a period of 24 months, patients with CKD G5 defined by KDOQI 2002 criteria, which underwent chronic hemodialysis three times per week and 4 hours duration per dialysis session, biochemical parameters were taken at the beginning of the dialysis session such as hemoglobin, parathyroid hormone, ferritin, plasma albumin, urea nitrogen, and serum electrolytes.

Analyzing demographical characteristics, laboratory variables, associated comorbidities and phase angle at $50 \mathrm{kHz}$ (PA50) measured by multi-frequency bioimpedance using a spectroscopic bioimpedance machine. (BCM-Fressenius Medical Care D GmbH). As a protocol measure in the institution, bioimpedance is performed when the patient is admitted to the renal unit classified as chronic kidney disease and monthly follow-up.

On a total of 104 patients.

\section{Inclusion criteria}

Patients prevalent in hemodialysis, more than 90 days undergoing dialysis, patients over the age of 18 years undergoing construction of a native arteriovenous fistula.

\section{Exclusion criteria}

Patients who underwent construction of a native arteriovenous fistula but didn't have their complete data registered patients who passed away during the data recollection period (Table 1).

The data and variables related to laboratory were obtained from clinical electronic registers corresponding to the period during the construction of the arteriovenous fistula, which was performed by a vascular surgeon experienced in the construction of vascular access destined for hemodialysis. Bioimpedance measurements were taken 


\section{9}

Patients in recruitment period, undergoing native AVF

construction by vascular surgeon for a period of 24 months

5

Excluded patients:

1 patient carrying pacemaker.

4 lost: 3 deaths, 1 lost tracking

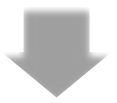

104

Patients meet inclusión criteria were undergone statistical analysis

Figure 2: Flow chart of study population.

by the nutrition group, according to specification of the dialysis center; prior to starting dialytic treatment with the patient a supine position, electrodes were placed on the arm and leg of the same side of the body; following recommendations made by its manufactures to assure standardization of all measurements. The $50 \mathrm{kHz}$ phase angle determinations were obtained through a data storage card included in the bioimpedance device and its incorporated program Fluid Manager Tool (FMT Fressenius Medical Care) and the electronic clinical register during the evaluation performed by the nutrition group prior to the surgical procedure. Bioimpedance measurements were made at the beginning and at one month with the sequential intake on 3 occasions and the average value were used (Table 2).

The maturation criterion used in the study was the use of the fistula with two needles in $75 \%$ of the sessions during a time interval of 4 weeks, with blood flow administered by the dialysis machine of $300 \mathrm{ml} /$ $\min$ (at least 4 continuous sessions) or $1.4 \mathrm{ktv}$ or a urea reduction rate of $70 \%$ (it was not possible to use ultrasound-Doppler measurements due to lack of equipment and economic deficit). During the followup, the moment of prior evaluation is documented by a nursing group the successful or not puncture of the fistula, considering a functional maturation when a fistula is successfully and effectively cannulated with a two-needle method which allows for dialytic treatment with adequate flow and pressures therefore a non-successful fistula is one that doesn't allow to be punctured, those that presented primary failure and those that required another vascular access for dialytic treatment within 12 weeks of its construction.

\section{Statistical analysis}

SPSS version 20.0 software was used for the statistical analysis of patients that presented primary failure in the process of functional maturation of the vascular access as primary result as was defined within the materials and methods section and its relation with the phase angle and serum albumin concentration.

The Kolmogorov Smirnov test was applied to define the distribution of variables; the parametric variables were represented as a mean average and standard deviation and non-parametric variable as median and interquartile ranges. Univariate logistic regression analysis was performed on variables of interest for predicting failure of vascular access. Including dependent variables which showed statistical significance for the subsequent multivariate analysis. Area under the curve was obtained and a cut-off point was determined with the highest sensibility and specificity for predicting failure of fistula by phase angle. Statistical significance was set at $\mathrm{P}<0.05$.

\section{Results}

Of 104 individuals followed during the development of this study a $32.2 \%$ achieved a successful maturation of the fistula which allowed for optimal dialytic treatment; in the remaining $67.8 \%$ of individual an adequate vascular access was not achieved, which is similar to data described in current literature. Of this last group the records of 73 male participants (70.2\%), 31 female participants (29.8\%) with a mean age of 62 years (SD: 14 years) 57.7\% of all patients were diabetic, $97.1 \%$ had hypertension, $42.3 \%$ with a background of peripheral artery disease. $92.5 \%$ of the studied population had a central venous catheter at the moment of construction of the arteriovenous fistula (Table 1).

Among the analytical variables presented were hemoglobin, ferritin and phase angle for males as well as females (Table 2); measurements were of frequency in terms of its median and respective standard deviations. For plasma albumin and parathyroid hormone its means and measurements of tendency were described in terms of interquartile ranges set at 25 and 75 respectively.

Univariate analysis for measurements of parathyroid hormone and ferritin did not show any association with the primary event evaluated. Similar findings were obtained for measurements of hemoglobin and body mass index.

Cut-off points were established for phase angle in female patients at 3.25 with a sensibility of $90 \%$ and specificity $53 \%$; and for males' phase angle at 3.58 for a sensibility of $84 \%$ and specificity of $52 \%$ with an area under the curve of 0.78 (Figure 3 ).

The binary logistic regression analysis for plasma and phase angle variable only showed statistical significance for the latter one in the evaluation of the primary objective (Table 3 ).

\section{Discussion}

As far as known, this is the first study designed to attempt establish if phase angle measurement through bioimpedance predicts maturation failure of arteriovenous fistulas. Within the studied population

Table 1: Demographic characteristics.

\begin{tabular}{|l|c|c|}
\hline \multicolumn{1}{|c|}{$\mathbf{n = 1 0 4}$} & Absolute frequency & Percentage \\
\hline Female & 73 & $70,2 \%$ \\
\hline Male & 31 & $29,8 \%$ \\
\hline Diabetes & 60 & $57,7 \%$ \\
\hline HTA & 101 & $97,1 \%$ \\
\hline PAD & 44 & $42,3 \%$ \\
\hline CVC & 99 & $95,2 \%$ \\
\hline
\end{tabular}

HTA: Arterial Hypertension, CVC: Central Venous Catheter, PAD: Peripheral Artery Disease. 


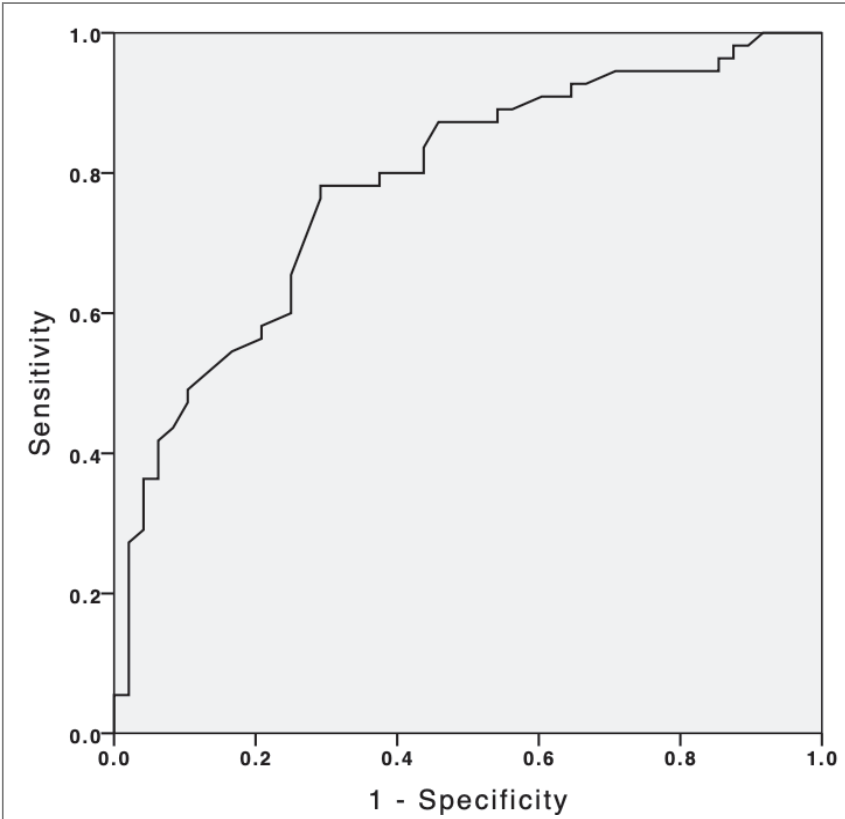

Figure 3: Phase angle from bioelectrical impedance.

Roc curve phase angle 0,78. Cut-off points were established for phase angle in female patients at 3.25 and for males' phase angle at 3.58.

Table 2: Analytical laboratory variables.

\begin{tabular}{|l|c|}
\hline \multicolumn{1}{|c|}{$\mathbf{n = 1 0 4}$} & Mean \pm SD \\
\hline Hb (mg/dl) & $10 \pm 1,83$ \\
\hline BMI & $23.1 \pm 4,2$ \\
\hline Age(years) & $62 \pm 14$ \\
\hline Ferritin (ng/ml) & $591 \pm 429$ \\
\hline Phase angle (F) & $3,57 \pm 1,07$ \\
\hline Phase angle(M) & $4,27 \pm 1,25$ \\
\hline PTH (nmol/l) RIQ & RIQ 25:157 RIQ 75:395 \\
\hline Albumin (gr/dl) RIQ & RIQ 25:3,79 RIQ 75:4,1 \\
\hline
\end{tabular}

HB: Hemoglobin, BMI: Body Mass Index, IQR: Interquartile Range, PTH: Parathyroid Hormone.

Table 3: Binary logistic regression for predicting failure of arteriovenous fistula.

\begin{tabular}{|l|c|c|}
\hline \multicolumn{1}{|c|}{ Variables } & OR (Cl 95) & P value \\
\hline Phase angle & $2,61(1,66-4,1)$ & 0.001 \\
\hline Albumin (gr/dl) & $3,31(0,30-30)$ & 0,34 \\
\hline
\end{tabular}

HB: Hemoglobin, BMI: Body Mass Index, PTH: Parathyroid Hormone.

we evaluated clinical characteristics, traditional variables, plasma albumin, and phase angle; this last one showed statistical significance within the evaluation of the primary objective (Table 4).

Failure of establishing vascular access remains a frequent cause of morbidity and elevated healthcare costs for patients undergoing hemodialysis; investigation of such determinants of vascular access maturation failure will have direct implications on the care these patients receive. Due to identifying patients at risk of vascular access failure being of capital importance, efforts must be directed towards the search of more objective ways of predicting; current guidelines
Table 4: Univariate analysis of predictors for primary failure in arteriovenous fistula.

\begin{tabular}{|l|c|c|}
\hline \multicolumn{1}{|c|}{ n 104 } & OR (Cl 95\%) & P value \\
\hline Hb (mg/dl) & $1,06(0,86-1,31)$ & 0,55 \\
\hline Ferritin (ng/ml) & $1,09(0,99-1.01)$ & 0,78 \\
\hline PTH(nmol/l) & $1,00(0,99-1,00)$ & 0,72 \\
\hline Albumin (gr/dl).log & $13,7(1,3-137,3)$ & 0,026 \\
\hline BMI & $1,04(0,95-1,14)$ & 0,368 \\
\hline Phase angle & $2,69(1,71-4,23)$ & 0,001 \\
\hline
\end{tabular}

HB: Hemoglobin, BMI: Body Mass Index, PTH: Parathyroid Hormone.

focusing on preventing vascular access failure seek to identify the patients with most risk factors, complete physical examination, use of biochemical parameters and adequate imaging resources.

Plasma albumin is one of the biochemical parameters used as a nutritional measure and a tool that allows the diagnosis of malnutrition or malnutrition or loss of caloric proteins and, like other published studies, it is correlated with worse results at the time of the construction of an arteriovenous fistula, but insufficient from a clinical point of view because despite the fact that plasma albumin is still measured in previous studies and in daily clinical practice, the results are scarce to try to predict the failure of arteriovenous fistulas.

In this study, it was shown that the bioimpedance phase angle is superior to albumin in predicting failure in arteriovenous fistulae, in addition, another advantage offered by the bioimpedance phase angle is its low cost, rapid operation, little technical difficulty and its non-functional nature. invasive qualify it as one of the recommended methods to estimate body composition and more in this population of patients with chronic kidney disease on hemodialysis where protein caloric loss is more accentuated with greater morbidity and mortality, and they use only albumin as a parameter.

Nutritional is insufficient because its result is subject to multiple variations due to the clinical conditions of these patients

That is why the phase angle becomes a useful tool not only as a predictor in the failure of arteriovenous fistulas but also as one of the most recommended methods at present to measure body composition and better discriminate nutritional status.

\section{Conclusions}

Our study suggests that the measurement of phase angle through multifrequency bioimpedance at $50 \mathrm{kHz}$, with cut-off values of 3.25 for female patients and 3.58 for male patients, it presents a significant association with adverse results in fistulas for hemodialysis in terms of primary failure and/or failure to achieve functional maturation; This finding could be explained by the relationship between the low phase angle and malnutrition in patients in dialysis as reported by other authors, Which lead us to infer that the poor nutritional status could be an independent risk factor for the development of adverse results after the construction of native hemodialysis fistulas.

Although our study is not designed to establish the role of specific mechanisms that explain the failure vascular access, if it allows for better selection of patients at the highest risk of failure in the pre-operative period order to establish interventions measures of potentially modifiable factors such as nutritional status and preserve the vascular beds of them in whom the risk fails to achieve maturation is high. 
We conclude that phase angle measured through bioimpedance, could be a useful tool to be considered in the identification of patients at risk of native fistula for dialysis maturation failure during the presurgical stage.

\section{Limitations}

Bioimpedance integrates measurements of various body segments with variable physical effects such as hydration status, fatty tissue percentage, lean tissue and percentage of total body water and given low phase angle values are associated with worse nutritional conditions and worse prognostics additional investigations are required that allow defining if malnutrition can behave as a new risk factor for adverse results related to fistulas to account for

To date, references test for prediction of access failure in the preoperative stage are lacking, so it was not possible to establish our groups of study without comparison; This work opens the possibility of future research to make use of the emergent tools and technologies for use by the vascular surgeon and nephrologists as a part of clinical daily practice for preoperative evaluation the patients in whom the construction of native hemodialysis fistulas is planned.

Difficulties in validation bioimpedance in different ethnic groups, variability in clinical conditions and abnormal hydration status have led to a large quantity of equations which can be of difficult interpretation and application within the context of the studied population.

\section{Funding}

Not applicable. Our manuscript does not contain any funding.

\section{Availability of Data and Materials}

The data and materials were all included in the manuscript.

\section{Authors' Contributions}

Jose Lucas Daza (JLD) is responsible for the writing of the manuscript. JLD performed the acquisition and interpretation of data and drafted and revised the manuscript. All authors read and approved the final manuscript.

\section{Ethics Approval and Consent to Participate}

Written informed consent was obtained from the patient for publication of this manuscript, and the accompanying images were additional at the bibliographic references. This study/report was approved by the Ethical Committee of the Fresenius Medical Care and was conducted in accordance with the National Law on the protection of personal data No 25.326 and the Declaration of Helsinki (Last version, Fortaleza 2013).

\section{Consent for Publication}

Written informed consent was obtained from the patient for publication of this manuscript, and the accompanying images were additional at the bibliographic references.

\section{Competing Interests}

The authors declare that they have no competing interests.

\section{References}

1. Pisoni RL, Arrinton CJ, Albert JM, Ethier J, Kimata N, et al. (2009) Facility hemodialysis vascular access use and mortality in countries participating in DOPPS: an instrumental variable analysis. Am J Kidney Dis. 53: 475-491.
2. Pietro R, Palmer SC, Oliver MJ, Quinn RR, MacRae JM, et al. (2013) Associations between hemodialysis access type and clinical outcomes: a systematic review. J Am Soc Nephrol 24: 465-473.

3. McGrogan DG, Maxwell AP, Khawaja AZ, Inston NG (2015) Current tools for prediction of arteriovenous fistula outcomes. Clin Kidney J 8: 282-289.

4. Bradbury BD, Fissell RB, Albert JM, Anthony MS, Critchlow CW, et al. (2007) Predictors of early mortality among incident US hemodialysis patients in the Dialysis Outcomes and Practice Patterns Study (DOPPS). Clin J Am Soc Nephrol 2: 89-99.

5. Malas MB, Canner JK, Hicks CW, Arhuidese IJ, Zarkowsky DS, et al. (2015) Trends in incident hemodialysis access and mortality. JAMA Surg 150: 441-448.

6. Vascular Access Work Group (2006) Clinical practice guidelines for vascular access. Am J Kidney Dis 48: S248-S273.

7. Wells AC, Fernando B, Butler A, Huguet E, Bradley JA, et al. (2005) Selective use of ultrasonographic vascular mapping in the assessment of patients before haemodialysis access surgery. $\mathrm{Br} \mathrm{J}$ Surg 92: 1439-1443.

8. Smith GE, Gohil R, Chetter IC (2012) Factors affecting the patency of arteriovenous fistula for dialysis access. J Vasc surg 55: 849-855.

9. Hod T, Desilva RN, Patibandla BK, Vin Y, Brown RS, et al. (2014) Factors predicting failure of AV "fistula first" policy in the elderly. Hemodial Int 18: 507-515.

10. Wong V, Ward R, Taylor J, Selvakumar S, How TV, et al. (1996) Factors associated with early failure of arteriovenous fistulae for haemodialysis access. Eur J Vasc Endovasc Surg 12: 207-213.

11. Lok CE, Allon M, Louise M, Oliver MJ, Shah H, et al. (2006) Risk equation determining unsuccessful cannulation events and failure to maturation in arteriovenous fistulas (REDUCE FTM I). J Am Soc Nephrol 17: 3204-3212.

12. Gracia-Iguacel C, González-Parra E, Barril-Cuadrado G, Sánchez R, Egido J, et al. (2014) Defining protein-energy wasting syndrome in chronic kidney disease: prevalence and clinical implications. Nefrologia 34: 507-519.

13. Maggiore Q, Nigrelli S, Cicarelli C, Grimaldi C, Rossi GA, et al. (1996) Nutritional and prognostic correlates of bioimpedance indexes in hemodialysis patients. Kidney Int 502: 2103-2108.

14. Louis $M$, Alexandrine $O$, Bendayan $M$, Trnkus $A$, Ouimet $M C$, et al. (2018) Phase Angle as Biomarker for Frailty and Postoperative Mortality: The BICS Study. J Am Heart Assoc 7: e008721.

15. Lopez-Gomez JM (2011) Evolution and applications of bioimpedance in managing chronic kidney disease. Nefrologia 31: 630-634.

16. Dumler F, Kilates $C(2000)$ Use of bioelectrical impedance techniques for monitoring nutritional status in patients on maintenance dialysis. J Ren Nutr 10: 116-124.

17. Nescolarde L, Picoli A, Roman A, Nuñez A, Morales R, et al. (2004) Bioelectrical impedance vector analysis in haemodialysis patients: relation between oedema and mortality. Physiol Meas 25: 12711280.

18. Ursula GK, Bosaeus I, De Lorenzo AD, Deurenberg P, Elia M, et al. (2004) Bioelectrical Impedance analysis--part I: Review of principles and methods. Clin Nutr 23: 1226-1243.

19. Rimsevicius L, Gincaite A, Vicka V, Sukackiene D, Pavinic J, at al. (2016) Malnutrition assessment in hemodialysis patients: Role of bioelectrical Impedance analysis phase angle. J Ren Nutr 26: 391395. 
20. Caravaca F, del Viejo CM, Villa J, Gallardo RM. Rocio, Ferreira F (2011) Hydration status assessment by multi-frecuency bioimpedance in patients with advanced chronic kidney disease. Nefrologia 31: 537544.

21. Fürstenberg A, Davenport A (2010) Comparison of multifrequency bioelectrical impedance analysis and dual-energy X-ray absorptiometry assessments in outpatient hemodialysis patients. Am J Kidney Dis 57: 123-129.

22. Ringaitiene D, Gineityte D, Vicka V, ZvirblisT, Norkiene I, et al. (2016) Malnutrition assessed by phase angle determines outcomes in lowrisk cardiac surgery patients. Clin Nutr 35: 1328-1332.
23. Shin JH, Kim CR, Park KH, Hwang JH, Kim SH (2017) Predicting clinical outcomes using phase angle as assessed by bioelectrical impedance analysis in maintenance hemodialysis patients. Nutrition 41: 7-13.

24. Premuzic V, Hudolin T, Pasini J, Zimak Z, Hauptman D, et al. (2018) Hypoproteinemia as a prognostic risk factor for arteriovenous fistula failure. Hemodial Int 22: 37-44.

25. Kordzadeh A, Tokidis E, Askari A, Hoff M, Panayiotopoulos Y (2017) The independent association of preoperative serum albumin on the functional maturation of radiocephalic arteriovenous fistulae. J Vasc Access 18: 148-152. 